\title{
Trace Elements in Soils around Coal Mines: Current Scenario, Impact and Available Techniques for Management
}

\author{
Prafulla Kumar Sahoo $^{1}$ - Sk. Md. Equeenuddin ${ }^{2}$ - M. A. Powell ${ }^{3}$
}

Published online: 29 January 2016

(C) Springer International Publishing AG 2016

\begin{abstract}
The present paper reviewed the average concentrations of trace elements in soils near coal mine areas from various cities/countries to provide a current global summary of this issue. The contamination of soils was assessed by using global reference materials and various contamination indices. The results show that the average concentrations of trace elements varied widely: As $(0.5-38.3 \mathrm{mg} / \mathrm{kg}), \mathrm{Mn}(86-3$, $700 \mathrm{mg} / \mathrm{kg}), \mathrm{Zn}(1.5-296 \mathrm{mg} / \mathrm{kg}), \mathrm{Cu}(0.5-110 \mathrm{mg} / \mathrm{kg}), \mathrm{Cr}$ (17.5-954 mg/kg), Ni (4.3-390 mg/kg), Pb (0.5-433 mg/kg), $\mathrm{Cd}(0.02-4.48 \mathrm{mg} / \mathrm{kg})$, and $\mathrm{Hg}(0.02-0.69 \mathrm{mg} / \mathrm{kg})$. Most of these values are higher than the average world background soils and upper continental crust (UCC). The geoaccumulation $\left(I_{g e o}\right)$ and contamination factor $(C F)$ indices show that the contamination levels of $\mathrm{Ni}$ and $\mathrm{Pb}$ are higher than other elements. The potential ecological risk index $\left(E_{i}\right)$ suggests a high risk from Cd, especially in the Tabagi River watershed in Brazil and Ledo coal mines in India, and $\mathrm{Hg}$, especially in Oltu (Turkey). The degree of contamination in$\operatorname{dex}\left(C_{d}\right)$ reveals that the soils from Barapukuria (Bangladesh), Ledo (India), Ptolemais-Amynteon (Greece), and the Tibagi River (Brazil) have a higher degree of contamination than
\end{abstract}

This article is part of the Topical Collection on Land Pollution

Prafulla Kumar Sahoo

prafulla.iitkgp@gmail.com

1 Vale Institute of Technology, Rua Boaventura da Silva 955, Nazaré, 66055-090 Belém, Pará, Brazil

2 Department of Mining Engineering, National Institute of Technology, Rourkela 769008, Odisha, India

3 Faculty of Agriculture, Life and Environmental Sciences, Department of Renewable Resources, University of Alberta, Edmonton, AB, Canada T6G 2R3 other sites. To control soil contamination, effective monitoring and legislative measures must be taken immediately. For the long term, various physical, chemical, and biological methods should be implemented. Although the selection of suitable methods is site-specific based on the extent and nature of contamination, in general, phytoremediation seems to be more beneficial and cost-effective than physical/chemical techniques.

Keywords Soil $\cdot$ Trace elements · Contamination $\cdot$ Pollution index $\cdot$ Remediation

\section{Introduction}

Coal is the most abundant fossil fuel on the earth and supplies about $75 \%$ of the total fuel resources [1]. It is the major source of energy production, contributing over $40 \%$ of the world's electricity generation (Figs. 1 and 2), and is used heavily in the steel industry [2]. China is the biggest coal-producing country, contributing nearly $45 \%$ of the world's total coal output, followed by the USA and India, contributing $14.5 \%$ and $6.8 \%$, respectively, of the world's total coal production [3]. Coal production has important economic and social benefits; however, it generates large amounts of coal mine spoils, and waste rocks which oxidize under atmospheric conditions and release metal-rich effluents to the surrounding environment. This causes serious problems to surrounding water-soil bodies [4-6]. Soils are the major sink for trace elements released into the environment because of their high metal-scavenging potential [7]. This, in turn, degrades the chemical and microbiological quality of soil $[8 \bullet, 9-11,12 \bullet]$, and subsequently creates a threat to humans and the ecosystem through direct contact with contaminated soils and the food chain because trace elements can be transferred from soil to plants and impact on 
Fig. 1 Percentage of coal used for electricity production worldwide [2]

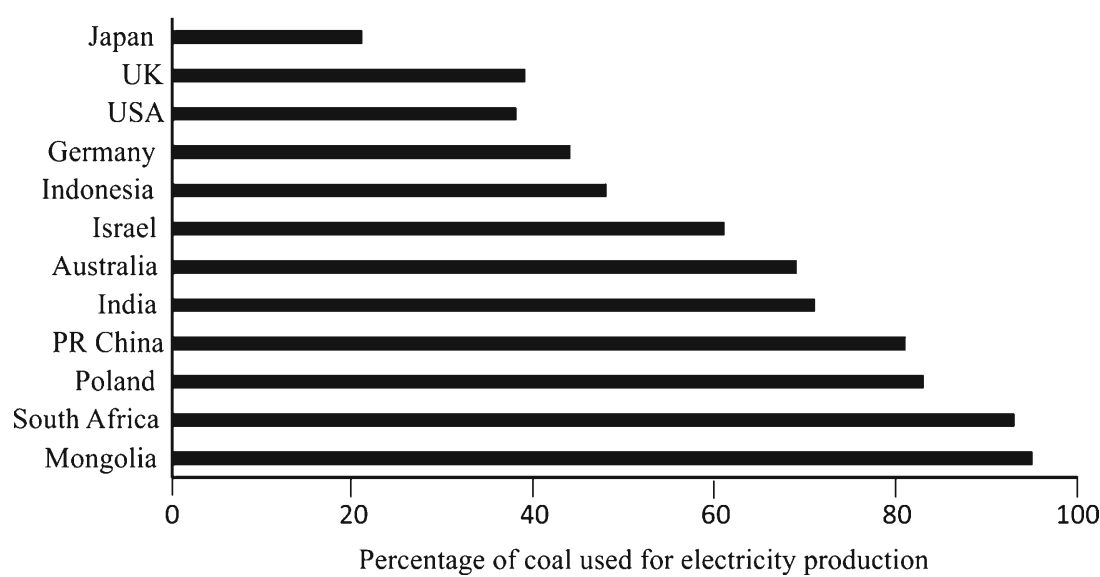

crop growth and food safety $[10,11,13]$. Thus, research on soil contamination around coal mine sites is receiving increasing attention in restoration of soil ecosystems and their sustainable use. There are several methods that have been widely used for evaluating trace element contamination in soils. The most commonly used pollution indices are: single element indices such as the geo-accumulation index $\left(I_{g e o}\right)$, the enrichment factor $(E F)$, the contamination factor $(C F)$, and ecological risk $\left(E_{i}\right)$; and multi-element indices such as the pollution load index $(P I L)$, the degree of contamination $\left(C_{d}\right)$, and Nemerow integrated pollution index (NIPI) [8・, 14-16]. Although each index has limitations, the combination of these approaches can be a good option for providing a more comprehensive and accurate assessment of trace element contamination. In the last few decades, several individual studies have been carried out investigating this issue around coal mines worldwide $[8 \bullet, 17,18]$. However, to date there has been no comprehensive review on these studies.

In the present paper, we review data on total concentrations of trace elements in soils nearby coal mines from various countries and evaluate their contamination status using global reference/background values and various contamination indices. This review thus provides a global scenario of soil contamination around coal mines. In addition, we discuss various physical/chemical/biological techniques available for

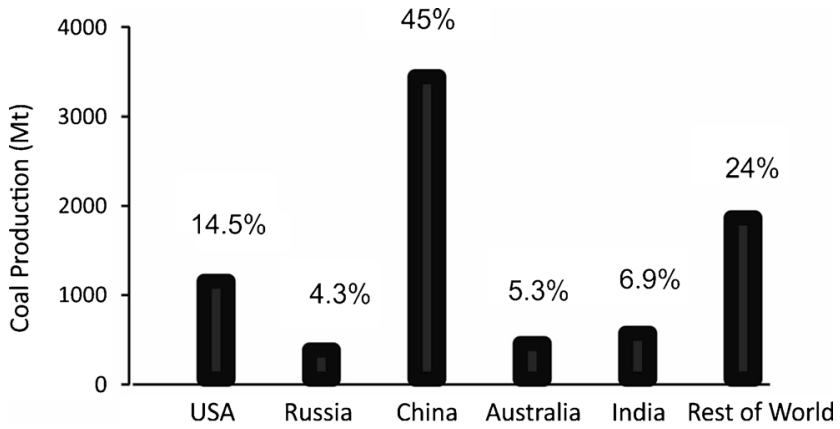

Fig. 2 Coal production (Mt) in the year 2011 worldwide; the percentage at the top of each bar represents the individual contribution relative to total production [3] remediation of metal-contaminated soils, which can be helpful for the selection of suitable strategies to employ for contaminated sites. The limitation of this review is that it is based on the availability of online literature and may not fully represent soil pollution in the vicinity of coal mines around the world. Furthermore, it is recognized that methodologies vary across these studies. However, these factors do not significantly affect the general assessment results because the research methods used are quite similar and are widely accepted by the scientific community.

\section{Calculation of Pollution Indices}

\section{Contamination Factor $(C F)$}

Contamination factor $(C F)$ was employed to assess the pollution potential of individual elements in soils. This is calculated using the following equation (Eq. 1) [19].

$C F=\frac{C_{\text {metal }}}{C_{\text {control }}}$

where $C_{\text {metal }}$ and $C_{\text {control }}$ are concentrations of metals in contaminated and background samples, respectively. In this study, world background soil was used as the control value. The level of contamination is classified according to the $C F$ values given in Table 1 [19].

\section{Geo-accumulation Index $\left(I_{g e o}\right)$}

The geo-accumulation index $\left(I_{g e o}\right)$ was introduced by Muller [20] to evaluate trace element contamination in sediments, and it is now widely used to determine soil contamination [21]. $I_{\text {geo }}$ is calculated as follows (Eq. 2):

$I_{\text {geo }}=\log _{2}\left(\frac{C_{\text {metal }}}{1.5 C_{\text {metal }(\text { control })}}\right)$ 
Table 1 Classification according to $(C F)$ for soils [19] contamination factor

\begin{tabular}{ll}
$C F$ value & Contamination level \\
\hline $\mathrm{CF}<1$ & Low \\
$1 \leq \mathrm{CF}<3$ & Moderate \\
$3 \leq \mathrm{CF}<6$ & Considerable \\
$\mathrm{CF} \geq 6$ & Very high
\end{tabular}

where $C_{\text {metal }}$ is the concentration of the metal in the studied sample and $C_{\text {metal (control) }}$ is the geochemical background value (world background soil). The factor 1.5 is the correction factor used to minimize the effect of possible variations in the background or control values which may be attributed to terrigenous effects [22]. The six classes of $I_{\text {geo }}$ index values are given in Table 2 [20].

\section{Degree of Contamination $\left(C_{d e g}\right)$}

The sum of contamination factors for all elements for a particular sampling site represents the contamination degree $\left(C_{d e g}\right)$. This is calculated using the following equation (Eq. 3) [19]:

$C_{d e g}=\sum_{i=1}^{n} C F$

where $\mathrm{n}$ is the number of analyzed pollutants, and $C F$ is the contamination factor. The classification of " $C_{d e g}$ " is given in Table 3.

\section{Modified Degree of Contamination $\left(m C_{d}\right)$}

The modified degree of contamination index $\left(m C_{d}\right)$ is the sum of all the contamination factors for a given set of pollutants by the number of analyzed pollutants. This is a modified form of the Hakanson equation [23] and was calculated as follows (Eq. 4):

$m C_{d}=\frac{\sum_{i=1}^{n} C F}{n}$

where $\mathrm{n}$ is the number of analyzed pollutants and $C F$ is the contamination factor, which is calculated based on Eq. 1. The classification of $m C_{d}$ values is given in Table 4 [23]

\section{Pollution Load Index (PLI)}

Pollution load index $(P L I)$ is used to determine the total metal contamination in soils [24]. This index is the geometric mean of the contamination factor $(C F)$ of different trace metals present in the study area. PLI is expressed as:

$$
P L I=\sqrt[n]{C F_{1} \times C F_{2} \times C F_{3} \ldots \ldots \times C F_{n}}
$$

Table 2 Index of geoaccumulation $\left(\mathrm{I}_{\mathrm{geo}}\right)$ for contamination levels in soil [20]

\begin{tabular}{lll}
\hline Class & Value & Soil quality \\
\hline 0 & $\mathrm{I}_{\text {geo }} \leq 0$ & Uncontaminated \\
1 & $0<\mathrm{I}_{\text {geo }}<1$ & Uncontaminated/moderately contaminated \\
2 & $1<\mathrm{I}_{\text {geo }}<2$ & Moderately contaminated \\
3 & $2<\mathrm{I}_{\text {geo }}<3$ & Moderately/strongly contaminated \\
4 & $3<\mathrm{I}_{\text {geo }}<4$ & Strongly contaminated \\
5 & $4<\mathrm{I}_{\text {geo }}<5$ & Strongly/extremely contaminated \\
6 & $5<\mathrm{I}_{\text {geo }}$ & Extremely contaminated \\
\hline
\end{tabular}

where $\mathrm{n}$ is the number of metals and $C F$ is the contamination factor. A $P L I$ value $>1$ indicates soil is polluted whereas a $P L I$ value $<1$ indicates no pollution [14].

\section{Nemerow Integrated Pollution Index (NIPI)}

A Nemerow integrated index (NIPI) was applied to determine the quality of the soil environment [25] and is defined as follows (Eq. 6):

$N I P I=\sqrt{\frac{P I_{\text {avg }}^{2}+P I_{\max }^{2}}{2}}$

where $P I=C$, and $P I_{\text {avg }}^{2}$ and $P I_{\max }^{2}$ are the maximum and average $P I$ value of each metal, respectively. The quality of soil was classified into five grades according to the NIPI index (Table 5) [25].

\section{Ecological Risk Factor}

An ecological risk factor $\left(E_{i}\right)$ quantitatively expresses the potential ecological risk of a single contaminant. This can be calculated using the equation [19]:

$E_{i}=T_{f} \times C F$

where $T_{f}$ is the toxic response for a given element, and $C F$ is the contamination factor, which is calculated based on Eq. 1. The toxic-response factors for common trace elements such as $\mathrm{Pb}, \mathrm{Cd}, \mathrm{As}, \mathrm{Cu}$, and $\mathrm{Zn}$ were 5, 30, 10, 5, and 1, respectively. The terminology used to describe the risk factor is given in Table 6.

Table 3 Different degrees of contamination $\left(\mathrm{C}_{\mathrm{deg}}\right)$ for soils [19]

\begin{tabular}{ll}
\hline Class & Degree of contamination level \\
\hline $\mathrm{C}_{\mathrm{deg}}<8$ & Low degree of contamination \\
$8 \leq \mathrm{C}_{\mathrm{deg}}<16$ & Moderate degree of contamination \\
$16 \leq \mathrm{C}_{\mathrm{deg}}<32$ & Considerable degree of contamination \\
$\mathrm{C}_{\mathrm{deg}}>32$ & Very high degree of contamination \\
\hline
\end{tabular}


Table 4 Different classes of modified degree of contamination $\left(\mathrm{mC}_{\mathrm{d}}\right)$ values for soils [23]

\begin{tabular}{ll}
\hline Class & Degree of contamination level \\
\hline $\mathrm{mC}_{\mathrm{d}}<1.5$ & Nil to very low degree of contamination \\
$1.5 \leq \mathrm{mC}_{\mathrm{d}}<2$ & Low degree of contamination \\
$2 \leq \mathrm{mC}_{\mathrm{d}}<4$ & Moderate degree of contamination \\
$4 \leq \mathrm{mC}_{\mathrm{d}}<8$ & High degree of contamination \\
$8 \leq \mathrm{mC}_{\mathrm{d}}<16$ & Very high degree of contamination \\
$16 \leq \mathrm{mC}_{\mathrm{d}}<32$ & Extremely high degree of contamination \\
$16 \leq \mathrm{mC}_{\mathrm{d}}<32$ & Ultra high degree of contamination \\
\hline
\end{tabular}

\section{Potential Ecological Risk Index (RI)}

The potential ecological risk index $(R I)$ was introduced in the same way as the degree of contamination, and was defined as the sum of all risk factors for metals in soils. This is defined as follows (Eq. 8):

$R I=\sum_{i=1}^{n} E_{i}$

where $E_{i}$ is the single index of ecological risk factor, and $\mathrm{n}$ is the number of the trace element species. The classification of potential ecological risk and the relevant terminology are listed in Table 7 [19].

\section{Results and Discussion}

\section{Trace Element Content in Soils around Coal Mines}

The concentrations of trace elements in soils nearby coal fields from various countries are given in Table 8 [26-46, 47•, 48-57]. In order to facilitate a comparative analysis, world background soils [58, 59], upper crustal abundance value (UCC) [60], and other reference soils (China, the USA, Spain, and Brazil) were used [61-64]. The degree of contamination of individual metals was determined with the help of $C F$ and $I_{\text {geo }}$ (Tables 3 and 9). Although some authors have considered the local

Table 5 Different classes of Nemerow integrated pollution index $(N I P I)$ values of soils [25]

\begin{tabular}{ll}
\hline Class & Degree of contamination level \\
\hline $\mathrm{NIPI} \leq 0.7$ & Non-pollution \\
$0.7<\mathrm{NIPI} \leq 1$ & Warning line of pollution \\
$1<\mathrm{NIPI} \leq 2$ & Low level of pollution \\
$2<\mathrm{NIPI} \leq 3$ & Moderate level of contamination \\
$\mathrm{NIPI}>3$ & High level of pollution \\
\hline
\end{tabular}

Table 6 The ecological risk coefficient $\left(\mathrm{E}_{\mathrm{i}}\right)$ of soils [19]

\begin{tabular}{ll}
\hline Value & Pollution degree \\
\hline $\mathrm{E}_{\mathrm{i}}<40$ & Low \\
$40 \leq \mathrm{E}_{\mathrm{i}}<80$ & Moderate \\
$80 \leq \mathrm{E}_{\mathrm{i}}<160$ & Considerable \\
$160 \leq \mathrm{E}_{\mathrm{i}}<320$ & High \\
$\mathrm{E}_{\mathrm{i}} \geq 320$ & Very high \\
\hline
\end{tabular}

background soils as the reference for calculation of pollution indices, such background values vary from place to place and are less suitable for a global assessment. As such, global reference materials, such as world background soil [58, 59], have been used. Concentration data and contamination status of each metal are discussed below.

\section{Arsenic (As)}

Arsenic is considered a class ' $\mathrm{A}$ ' human carcinogen element in the USA [67], and therefore has generated considerable global attention to the environment and public health. The average As concentrations in surface soil in the vicinity of coal mines varied widely from 0.5 to $38.3 \mathrm{mg} / \mathrm{kg}$, and most of these values were higher than the respective values of UCC (Table 1). Concentrations in soils from Dingji (China), Barapukuria (Bangladesh), Ptolemais (Greece), Douro (Portugal), and Tula (Russia) exceeded those from world background soils, and also exceeded the values in US baseline soil [62], China reference soils [61], and the threshold value $(12 \mathrm{mg} / \mathrm{kg}) \mathrm{set}$ by CCME (Canadian Council of Ministers of the Environment). The highest average As value in soil was found at Douro coalfield in Portugal, which was the only site that exceeded the threshold value $(29 \mathrm{mg} / \mathrm{kg}$ ) for clean soil recommended by the Dutch Ministry, suggesting the need for prompt remediation of As contamination. The main source of As in soils is its parent material, but As accumulation in urban environments is most likely due to combustion of fossil fuels, particularly coal and mining activities [68].

The $I_{\text {geo }}$ value of As was lower compared to other metals, and ranges from -5.9 to 1.16 (Table 3). The maximum value, 1.16, was from Douro coalfield,
Table 7 The potential ecological risk coefficient (RI) of soils [19]

\begin{tabular}{ll}
\hline Value & Risk potential \\
\hline $\mathrm{RI}<50$ & Low \\
$50 \leq \mathrm{RI}<100$ & Moderate \\
$100 \leq \mathrm{RI}<200$ & High \\
$\mathrm{RI} \geq 200$ & Significantly very high
\end{tabular}


Table 8 Average concentrations $(\mathrm{mg} / \mathrm{kg}$ ) of trace elements in coal-mine soils from various countries compared with global reference values and other reference soils

\begin{tabular}{|c|c|c|c|c|c|c|c|c|c|c|c|c|}
\hline Location & Soil type & No. & As & $\mathrm{Mn}$ & $\mathrm{Zn}$ & $\mathrm{Cu}$ & $\mathrm{Cr}$ & $\mathrm{Ni}$ & $\mathrm{Pb}$ & $\mathrm{Cd}$ & $\mathrm{Hg}$ & Reference \\
\hline Antaibao, China & Reclaimed soil & - & - & - & - & 31.16 & 59.4 & - & 9.72 & 0.09 & 0.1 & [26] \\
\hline Fushun, China & Reclaimed soil & 3 & - & 142.3 & 46.3 & 16.23 & 42.77 & - & - & 0.42 & - & [27] \\
\hline Heidaigou, China & Reclaimed soil & - & - & - & 56.2 & 18.06 & 73.8 & 27.88 & 12.36 & 0.09 & 0.03 & [28] \\
\hline Laohutai, China & Reclaimed soil & - & - & - & 65.6 & 34.33 & 72.49 & - & - & 0.02 & - & [27] \\
\hline Pindingshan, China & Reclaimed soil & - & - & - & 39.9 & 38.99 & 594.1 & 19.2 & 61 & 1.3 & - & [29] \\
\hline Pingshuo, China & Reclaimed soil & - & - & - & - & - & 40.92 & - & 23 & 0.21 & 0.02 & [30] \\
\hline Xuzhou, China & Reclaimed soil & - & - & - & 130.9 & 52.3 & 73.4 & - & 47.4 & 3.2 & 0.03 & [31] \\
\hline Huainan, China & Reclaimed soil & - & - & - & 90.2 & 38.2 & 213.5 & 40 & 40 & 1.4 & 0.04 & [32] \\
\hline Datong, China & Reclaimed soil & - & 3.82 & - & - & 44.75 & - & - & 44.75 & 0.31 & 0.205 & [33] \\
\hline Panyi, China & Reclaimed soil & - & 6.44 & - & - & 40.28 & - & - & 52.93 & 0.43 & 0.126 & [33] \\
\hline Xinzhuangzi, China & Reclaimed soil & - & 5.86 & - & - & 43.52 & - & - & 42.58 & 0.51 & 0.177 & [33] \\
\hline Baodian, China & Reclaimed soil & 12 & & - & 101.8 & 30.9 & - & - & 37.78 & 1.97 & - & [34] \\
\hline Heidaigou, China & Reclaimed soil & 10 & 8.89 & - & 56.7 & 17 & 66 & 27.3 & 12.2 & 0.06 & 0.02 & [35] \\
\hline Huabei, China & & 45 & 8.47 & - & 69.3 & 19.8 & - & - & - & - & - & [36] \\
\hline Jilin Province, China & Top soil & - & - & - & 65.4 & 21.5 & 69.6 & - & 20.9 & 0.15 & - & [37] \\
\hline Dingji, China & Reclaimed soil & 8 & 17.6 & - & 46.5 & 30 & 41.1 & - & 23.8 & 0.13 & - & [38] \\
\hline Guizhou, China & Mine-impacted soil & - & - & - & 135.8 & - & - & - & 42.4 & - & - & [39] \\
\hline Raniganj, India & Native soil & 8 & - & 462.3 & 44.3 & 12.2 & 46.7 & 26.23 & 8.19 & 0.955 & - & [40] \\
\hline Surat, India & Agricultural soil & 8 & - & - & 108.3 & 110.4 & 37.9 & 65.5 & 17.8 & 1.56 & - & [41] \\
\hline Raniganj, India & Top soil & 3 & - & 86 & 148.6 & 47.3 & 240 & 124.6 & - & - & - & [42] \\
\hline Sonepur Bazari, India & Opencast mine-impacted soil & 32 & - & 3.96 & 947 & 27 & 98 & 34 & 27.3 & 0.012 & - & [43] \\
\hline Jaintia, India & AMD contaminated soil & 18 & - & 232 & 53.9 & 22.9 & 143 & 38.4 & 23 & 1.53 & - & [44] \\
\hline Makum, India & AMD contaminated soil & 11 & - & 399 & 63.4 & 22.7 & 202 & 83 & 13.9 & 1.32 & - & [45] \\
\hline Ledo, Tinsukia, India & Mine-impacted soil & - & - & - & & - & 112 & 87.5 & 183 & 2.6 & - & [46] \\
\hline Gangreung, Korea & AMD contaminated soil & - & - & - & 87.3 & 41 & 35.8 & 42.6 & 32.9 & 1.1 & - & [17] \\
\hline Barapukuria, Bangladesh & AMD contaminated soil & 30 & 17.5 & 1886 & 296 & - & - & - & 433 & - & - & {$[8 \bullet]$} \\
\hline Barapukuria, Bangladesh & Paddy soils & - & 22.4 & 1048 & 160 & 29 & 107 & 98.5 & 188.6 & - & - & [18] \\
\hline Tibagi River watershed, Brazil & Regular soils & - & - & 3700 & & - & - & - & - & 4.48 & - & {$[47 \cdot]$} \\
\hline Oltu, Turkey & & 19 & - & - & 35.9 & 23.4 & 135.6 & 59.8 & 34.2 & 0.03 & 0.69 & [48] \\
\hline Ptolemais, Greece & & 101 & 12.3 & - & - & - & 17.5 & 10.1 & - & - & - & [49] \\
\hline Ptolemais-Amynteon, Greece & Regular soil & - & 9.3 & - & 94.5 & 45.7 & 522.5 & 390 & 64.2 & 0.25 & - & [50] \\
\hline Ptolemais-Amynteon, Greece & Reclamed soil & - & 6.25 & - & 56 & 32.5 & 205.8 & 137 & 61.3 & 0.25 & - & {$[50]$} \\
\hline Douro, Portugal & Waste-impacted soil & 3 & 38.3 & 139 & 57 & 36.5 & 92.3 & 21.4 & 30.8 & 0.2 & - & [51] \\
\hline Tula, Moscow, Russia & & 134 & 17.4 & - & 164.5 & - & - & - & - & - & - & [52] \\
\hline Palapye, Botswana & Mine soil & 27 & 9.8 & - & 65 & 35.4 & 125.2 & 47.3 & 22.8 & - & - & [53] \\
\hline Smolnica, Southern Polland & Reclaimed soil, organic rich & - & - & - & 142 & 18 & - & - & 39.9 & 1.65 & - & [54] \\
\hline Emalahleni, South Africa & Mine-impacted soil & 693 & 0.5 & - & 36 & 22 & 419 & 20 & 19 & - & - & [55] \\
\hline Okaba, Nigeria & Mine-impacted soil & 11 & - & - & 1.5 & 0.5 & - & 4.3 & 0.5 & 0.6 & - & [56] \\
\hline Pokrok, Czech Republic & Reclaimed soil & 103 & 4.48 & 599 & 25.2 & 13.7 & - & - & 18.4 & 0.27 & - & [57] \\
\hline Minimum & & & 0.5 & 86 & 1.5 & 0.5 & 17.5 & 4.3 & 0.5 & 0.02 & 0.012 & \\
\hline Maximum & & & 38.3 & 3,700 & 296 & 110.4 & 523 & 390 & 433 & 4.48 & 0.69 & \\
\hline World background soil & & & 11.4 & 571 & 67.8 & 28.2 & 70.9 & 17.8 & 28.4 & 0.49 & $0.06^{*}$ & {$[58,59 *]$} \\
\hline Upper continental crust & & & 2 & 900 & 52 & 14 & 35 & 19 & 17 & 0.102 & 0.056 & {$[60]$} \\
\hline Reference soil, China & & & 11.2 & - & 74 & 23 & 61 & 27 & 27 & 0.097 & 0.065 & [61] \\
\hline Reference soil, USA & & & 5.5 & 380 & 55 & 21 & 41 & 15 & 17 & - & 0.05 & [62] \\
\hline Reference soil, Spain & & & - & - & 192 & - & 73 & 43 & 30 & 0.3 & - & [63] \\
\hline Reference soil, Brazil & & & - & - & 59.9 & 35.1 & 40.3 & 13.2 & 17 & 0.5 & - & [64] \\
\hline \multicolumn{3}{|c|}{ Target value, Dutch Ministry of Housing } & 29 & - & 140 & 36 & 100 & 35 & 85 & 0.8 & 0.3 & [65] \\
\hline \multicolumn{3}{|c|}{ Soil quality for agricultural soil, Canada } & 12 & - & 200 & 63 & 64 & 50 & 70 & 1.4 & 6.6 & [66] \\
\hline
\end{tabular}

No. number of samples, $\mathrm{As}$ arsenic, $\mathrm{Mn}$ manganese, $\mathrm{Zn}$ zinc, $\mathrm{Cu}$ copper, $\mathrm{Cr}$ chromium, $\mathrm{Ni}$ nickel, $\mathrm{Pb}$ lead, $\mathrm{Cd}$ cadmium, $\mathrm{Hg}$ mercury - indicates data not available

* indicates the Hg value cited from reference [59], while rests are cited from reference [60] 
Table 9 Contamination factor $(C F)$, Nemerow integrated pollution index (NIPI), integrated pollution index (IPL), degree of contamination $\left(\mathrm{C}_{\mathrm{deg}}\right)$, and modified degree of contamination $\left(\mathrm{m}_{\mathrm{d}}\right)$ of trace elements in coal-mine soils collected from various cities/countries

\begin{tabular}{|c|c|c|c|c|c|c|c|c|c|c|c|c|c|c|c|}
\hline \multirow[b]{2}{*}{ Location } & \multirow[b]{2}{*}{ Soil type } & \multicolumn{9}{|l|}{$C F$} & \multirow[b]{2}{*}{ NIPI } & \multirow[b]{2}{*}{ PLI } & \multirow[b]{2}{*}{$\mathrm{C}_{\mathrm{deg}}$} & \multirow[b]{2}{*}{${ }_{\mathrm{m}} \mathrm{C}_{\mathrm{d}}$} & \multirow[b]{2}{*}{ Reference } \\
\hline & & As & $\mathrm{Mn}$ & $\mathrm{Zn}$ & $\mathrm{Cu}$ & $\mathrm{Cr}$ & $\mathrm{Ni}$ & $\mathrm{Pb}$ & $\mathrm{Cd}$ & $\mathrm{Hg}$ & & & & & \\
\hline Antaibao, China & Reclaimed soil & - & - & - & 1.10 & 0.84 & - & 0.34 & 0.18 & 1.67 & 1.12 & 0.63 & 4.14 & 0.83 & {$[26]$} \\
\hline Fushun, China & Reclaimed soil & - & 0.25 & 0.68 & 0.58 & 0.60 & - & - & 0.86 & - & 0.88 & 0.67 & 2.72 & 0.68 & {$[27]$} \\
\hline Heidaigou, China & Reclaimed soil & - & - & 0.83 & 0.64 & 1.04 & 1.57 & 0.44 & 0.18 & 0.50 & 1.07 & 0.62 & 5.20 & 0.74 & {$[28]$} \\
\hline Laohutai, China & Reclaimed soil & - & - & 0.97 & 1.22 & 1.02 & - & & 0.04 & - & 1.01 & 0.47 & 3.25 & 0.81 & {$[27]$} \\
\hline Pindingshan, China & Reclaimed soil & - & - & 0.59 & 1.38 & 8.38 & 1.08 & 2.16 & 2.65 & - & 2.35 & 1.87 & 16.25 & 2.71 & [29] \\
\hline Pingshuo, China & Reclaimed soil & - & - & & & 0.58 & - & 0.82 & 0.43 & 0.33 & 0.82 & 0.51 & 2.15 & 0.54 & {$[30]$} \\
\hline Xuzhou, China & Reclaimed soil & - & - & 1.93 & 1.85 & 1.04 & - & 1.68 & 6.53 & 0.50 & 2.10 & 1.65 & 13.53 & 2.26 & {$[31]$} \\
\hline Huainan, China & Reclaimed soil & - & - & 1.33 & 1.35 & 3.01 & 2.25 & 1.42 & 2.86 & 0.67 & 1.56 & 1.65 & 12.89 & 1.84 & {$[32]$} \\
\hline Datong, China & Reclaimed soil & 0.34 & - & - & 1.59 & - & - & 1.59 & 0.63 & 3.42 & 1.57 & 1.13 & 7.56 & 1.51 & {$[33]$} \\
\hline Panyi, China & Reclaimed soil & 0.56 & - & - & 1.43 & - & - & 1.88 & 0.88 & 2.10 & 1.32 & 1.23 & 6.85 & 1.37 & {$[33]$} \\
\hline Xinzhuangzi, China & Reclaimed soil & 0.51 & - & - & 1.54 & - & - & 1.51 & 1.04 & 2.95 & 1.49 & 1.30 & 7.56 & 1.51 & {$[33]$} \\
\hline Baodian, China & Reclaimed soil & - & - & 1.50 & 1.10 & - & - & 1.34 & 4.02 & - & 1.73 & 1.73 & 7.96 & 1.99 & {$[34]$} \\
\hline Heidaigou, China & Reclaimed soil & 0.78 & - & 0.84 & 0.60 & 0.93 & 1.53 & 0.43 & 0.12 & 0.33 & 1.06 & 0.56 & 5.57 & 0.70 & {$[35]$} \\
\hline Huabei, China & & 0.74 & - & 1.02 & 0.70 & - & - & - & - & - & 0.96 & 0.81 & 2.47 & 0.82 & {$[36]$} \\
\hline Jilin Province, China & Top soil & - & - & 0.96 & 0.76 & 0.98 & - & 0.74 & 0.31 & - & 0.93 & 0.70 & 3.76 & 0.75 & {$[37]$} \\
\hline Dingji, China & Reclaimed soil & 1.54 & - & 0.69 & 1.06 & 0.58 & - & 0.84 & 0.27 & - & 1.09 & 0.73 & 4.98 & 0.83 & {$[38]$} \\
\hline Guizhou, China & Mine-impacted soil & - & - & 2.00 & - & - & - & 1.54 & - & - & 1.37 & 1.74 & 3.51 & 1.75 & [39] \\
\hline Raniganj, India & Native soil & - & 0.81 & 0.65 & 0.43 & 0.66 & 1.47 & 0.29 & 1.95 & - & 1.19 & 0.74 & 6.27 & 0.90 & {$[40]$} \\
\hline Surat, India & Agricultural soil & - & & 1.60 & 3.91 & 0.53 & 3.68 & 0.63 & 3.18 & - & 1.76 & 1.71 & 13.54 & 1.93 & {$[41]$} \\
\hline Raniganj, India & Top soil & - & 0.15 & 2.19 & 1.68 & 3.39 & 7.00 & - & - & - & 2.22 & 1.67 & 14.40 & 2.88 & {$[42]$} \\
\hline Sonepur Bazari, India & Opencast mine soil & 0.34 & 1.68 & 0.69 & 0.95 & 1.38 & 1.91 & 0.96 & 0.024 & - & 1.2 & 0.68 & 7.94 & 1.13 & {$[43]$} \\
\hline Jaintia, India & AMD contaminated soil & - & 0.41 & 0.79 & 0.81 & 2.02 & 2.16 & 0.82 & 3.12 & - & 1.51 & 1.16 & 10.13 & 1.45 & {$[44]$} \\
\hline Makum, India & AMD contaminated soil & - & 0.70 & 0.94 & 0.80 & 2.85 & 4.66 & 0.49 & 2.69 & - & 1.81 & 1.37 & 13.14 & 1.88 & {$[45]$} \\
\hline Ledo, Tinsukia, India & Mine-impacted soil & - & - & - & - & 1.58 & 4.92 & 6.48 & 5.3 & - & 2.35 & 4.04 & 18.29 & 4.57 & {$[46]$} \\
\hline Gangreung, Korea & AMD contaminated soil & - & - & 1.29 & 1.45 & 0.50 & 2.39 & 1.17 & 2.24 & - & 1.40 & 1.29 & 9.05 & 1.29 & {$[17]$} \\
\hline Barapukuria, Bangladesh & AMD contaminated soil & 1.54 & 3.30 & 4.37 & - & - & - & 15.35 & - & - & 3.28 & 4.29 & 24.56 & 6.14 & {$[8 \bullet]$} \\
\hline Barapukuria, Bangladesh & Paddy soils & 1.96 & 1.84 & 2.36 & 1.03 & 1.51 & 5.53 & 6.69 & - & - & 2.20 & 2.42 & 20.92 & 2.99 & {$[18]$} \\
\hline Tibagi River watershed, Brazil & Regular soils & - & 6.48 & - & - & - & - & - & 9.14 & - & 3.01 & 7.70 & 15.62 & 7.81 & {$[47 \cdot]$} \\
\hline Oltu, Turkey & & - & - & 0.53 & 0.83 & 1.91 & 3.36 & 1.21 & 0.06 & 11.50 & 2.67 & 0.77 & 19.41 & 2.77 & {$[48]$} \\
\hline Ptolemais, Greece & & 1.08 & - & - & - & 0.25 & 0.57 & - & - & - & 0.92 & 0.53 & 1.89 & 0.63 & [49] \\
\hline Ptolemais-Amynteon, Greece & Regular soil & 0.82 & - & 1.39 & 1.62 & 7.37 & 21.91 & 2.28 & 0.51 & - & 3.68 & 2.30 & 35.90 & 5.13 & {$[50]$} \\
\hline Ptolemais-Amynteon, Greece & Reclamed soil & 0.55 & - & 0.83 & 1.15 & 2.90 & 7.70 & 2.17 & 0.51 & - & 2.23 & 1.44 & 15.81 & 2.26 & {$[50]$} \\
\hline Douro, Portugal & Waste-impacted soil & 3.36 & 0.24 & 0.84 & 1.29 & 1.30 & 1.20 & 1.09 & 0.41 & - & 1.51 & 0.93 & 9.74 & 1.22 & {$[51]$} \\
\hline Tula, Moscow, Russia & & 1.53 & - & 2.43 & - & - & - & - & - & - & 1.48 & 1.92 & 3.95 & 1.98 & {$[52]$} \\
\hline Palapye, Botswana & Mine soil & 0.86 & - & 0.96 & 1.26 & 1.77 & 2.66 & 0.81 & - & - & 1.42 & 1.26 & 8.31 & 1.38 & {$[53]$} \\
\hline Smolnica, Southern Polland & Reclaimed soil, organic & & - & 2.9 & 0.64 & - & - & 1.41 & 3.37 & - & 1.6 & 1.45 & 7.5 & 1.88 & {$[54]$} \\
\hline Emalahleni, South Africa & Mine soil & 0.04 & - & 0.53 & 0.78 & 5.9 & 1.12 & 0.67 & & - & 1.93 & 0.66 & 9.06 & 1.5 & {$[55]$} \\
\hline Okaba, Nigeria & Mine soil & & - & 0.02 & 0.02 & - & 0.24 & 0.02 & 1.22 & - & 0.87 & 0.07 & 1.5 & 0.25 & {$[56]$} \\
\hline Pokrok, Czech Republic & Reclaimed soil & 0.39 & 1.05 & 0.37 & 0.49 & - & - & 0.65 & 0.55 & - & 0.9 & 0.55 & 3.5 & 0.58 & {$[57]$} \\
\hline Minimum & & 0.04 & 0.15 & 0.02 & 0.02 & 0.25 & 0.24 & 0.02 & 0.02 & 0.33 & 0.82 & 0.07 & 1.52 & 0.25 & \\
\hline Maximum & & 3.36 & 6.48 & 4.37 & 3.91 & 8.38 & 21.91 & 15.35 & 9.14 & 11.50 & 3.68 & 7.70 & 35.90 & 7.81 & \\
\hline
\end{tabular}

No. number of samples, $\mathrm{As}$ arsenic, $\mathrm{Mn}$ manganese, $\mathrm{Zn}$ zinc, $\mathrm{Cu}$ copper, $\mathrm{Cr}$ chromium, $\mathrm{Ni}$ nickel, $\mathrm{Pb}$ lead, $\mathrm{Cd}$ cadmium, $\mathrm{Hg}$ mercury

- indicates data not available

Portugal, which indicates moderate contamination, while the rest are uncontaminated. Similarly, the $C F$ of As was low and varied from 0.04 to 3.36 (Table 9), confirming most soils have low to moderate As contamination. The highest $C F$ value was found in Douro, Portugal, similar to the $I_{g e o}$ values, indicating considerable contamination. 


\section{Cadmium (Cd)}

Cadmium is a toxic metal and an environmental hazard. The average $\mathrm{Cd}$ concentration in surface soils around the coal mine sites varies from 0.02 to $4.48 \mathrm{mg} / \mathrm{kg}$ (Table 8). Most of the soils values, except from Heidaigou and Laohuti (China) and Sonepur Bazari (India), are higher than the UCC and China reference soils. Compared to the world average soil, 14 out of 34 sites exceeded the average values of $\mathrm{Cd}$. The highest average concentration was reported in the surface soil samples from the Tibagi River watershed in Brazil. The target value of Cd for clean soil is $1 \mathrm{mg} / \mathrm{kg}$ of soil [65], which is exceeded in soils from Pindingshan, Xuzhou, Huainan, and Boadian in China; Jaintia and Makum in India; and Gangreung in Korea.

The $I_{g e o}$ values of $\mathrm{Cd}$ in studied soils varies from -5.9 to 2.64 (Table 10), indicating uncontaminated to moderatestrong contamination. The maximum values from the Tibagi River, Brazil and Xuzhoiu, China show moderate-strong contamination, with the rest having moderate to low contaminated categories. The $C F$ classified the soils as having low to very high contamination with respect to $\mathrm{Cd}$. The high $I_{g e o}$ was consistent with high $C F$ values.

\section{Chromium (Cr)}

The average $\mathrm{Cr}$ concentrations in the surface soils varied between 17.7 and $523 \mathrm{mg} / \mathrm{kg}$, and most soils exceeded the world soil average and UCC values (Table 8). The target value for clean soil and the intervention value for soil remediation as established by VROM are $100 \mathrm{mg} / \mathrm{kg}$ and $380 \mathrm{mg} / \mathrm{kg}$ [65], respectively. This indicates the average concentrations of soil from Greece exceeded this limit, implying this area needs to be promptly managed for $\mathrm{Cr}$ contamination. The remaining cities have sustainable $\mathrm{Cr}$ content in soil quality levels.

The obtained $I_{g e o}$ values vary from -2.6 to 2.48 (Table 10), which can be categorized as uncontaminated to moderately contaminated. While the $C F$ varies from 0.25 to 8.35 (Table 9), soils from Ptomemain in Greece indicate very high contamination.

\section{Nickel (Ni)}

The average Ni concentration varies from 4.3 to $390 \mathrm{mg} / \mathrm{kg}$. Most of the soils exceeded the average value of the world background soil and UCC (Table 8). Most of sites, except Okaca (Nigeria) and Ptolemais (Greece), also exceeded the $\mathrm{Ni}$ values compared to the US reference soil $(15 \mathrm{mg} / \mathrm{kg})$, while a total of 13 sites have higher values than the reference soil of China $(27 \mathrm{mg} / \mathrm{kg})$. The target and intervention values for Ni established by the Dutch are 35 and $210 \mathrm{mg} / \mathrm{kg}$, respectively [65]. Soils from Greece have an average value of $390 \mathrm{mg} / \mathrm{kg}$, which is higher than the target value, suggesting that remediation is needed.
In studied soils, $I_{\text {geo }}$ values range from -2.63 to 3.87 (Table 10). The regular soil from Ptolemais, Greece shows strong contamination, while reclaimed soil from this coal field, along with soil from Raniganj coalfield, showed moderate to strong contamination, while the rest are characterized as being uncontaminated to moderately contaminated. The $C F$ factor varies from 0.24 to 21.9 (Table 9); the highest value for soil from Ptolemais-Amynteon (Greece) shows very high contamination, and this soil also had the highest $I_{g e o}$ values.

\section{Zinc (Zn)}

The average $\mathrm{Zn}$ concentrations ranged from 1.5 to $296 \mathrm{mg} / \mathrm{kg}$, and the highest concentration was reported in the soils of Barapukuria coal mine, Bangladesh (Table 8). Most of the sites exceed the level of world background soils and UCC. However, all studies were below the intervention limit $(720 \mathrm{mg} / \mathrm{kg}$ ), which was proposed by VROM [65]. VROM proposed a $\mathrm{Zn}$ concentration of $140 \mathrm{mg} / \mathrm{kg}$ as the limit of sustainable soil quality, but the average concentrations of Raniganj, Barapukuria, and Tula coal mine regions are higher than the Dutch limit, indicating $\mathrm{Zn}$ contamination in these areas.

The $I_{\text {geo }}$ values of $Z n$ vary from -6.07 to 1.55 (Table 10 ), indicating low to moderate contamination. The soils from Barapukuria only show moderate contamination, while the remaining soils are classified as uncontaminated. This is consistent with the $C F$ value (Table 9).

\section{Copper (Cu)}

The average $\mathrm{Cu}$ concentrations in surface soils from various sites ranged from 0.5 to $110 \mathrm{mg} / \mathrm{kg}$ (Table 8). Except for the soils from Raniganj, Okaba, and Pokrok, the soils studied exceeded the average concentration of UCC. Compared to the world background soils, eight cities out of the total are higher than the limit; however, soils from Surat, Ranigang, and Gengreung have greater values than the target value of $36 \mathrm{mg} / \mathrm{kg}$ established by VROM [65].

The $I_{\text {geo }}$ values of $\mathrm{Cu}$ vary from -6.4 to 1.38 (Table 10), soil from Surat, India shows moderate contamination, while other soils fall under uncontaminated categories. Similarly, higher $C F$ values in soil from Surat indicate moderate contamination.

\section{Lead $(\mathbf{P b})$}

Lead is a major environmental contaminant in miningimpacted soils. The average $\mathrm{Pb}$ concentration varies from 0.5 to $433 \mathrm{mg} / \mathrm{kg}$, and most soils exceeded the respective UCC value, except for four soils (Table 8). Of the total cases reviewed, around $50 \%$ of soils exceeded the world background soil levels and reference 
Table 10 Geo-accumulation $\left(\mathrm{I}_{\mathrm{geo}}\right)$ values in soils collected from various sites around coal mines worldwide

\begin{tabular}{|c|c|c|c|c|c|c|c|c|c|c|c|}
\hline Locations & Soil Type & As & $\mathrm{Mn}$ & $\mathrm{Zn}$ & $\mathrm{Cu}$ & $\mathrm{Cr}$ & $\mathrm{Ni}$ & $\mathrm{Pb}$ & $\mathrm{Cd}$ & $\mathrm{Hg}$ & Reference \\
\hline Antaibao, China & Reclaimed soil & - & - & - & -0.44 & -0.84 & - & -2.13 & -3.00 & 0.15 & {$[26]$} \\
\hline Fushun, China & Reclaimed soil & - & - & -1.13 & -1.38 & -1.31 & - & - & -0.78 & - & [27] \\
\hline Heidaigou, China & Reclaimed soil & - & - & -0.85 & -1.23 & -0.53 & 0.06 & -1.79 & -3.00 & -1.58 & {$[28]$} \\
\hline Laohutai, China & Reclaimed soil & - & - & -0.63 & -0.30 & -0.55 & - & - & -5.17 & - & [27] \\
\hline Pindingshan, China & Reclaimed soil & - & - & -1.34 & -0.12 & 2.48 & -0.48 & 0.52 & 0.85 & - & [29] \\
\hline Pingshuo, China & Reclaimed soil & - & - & - & - & -1.38 & - & -0.89 & -1.78 & -2.17 & {$[30]$} \\
\hline Xuzhou, China & Reclaimed soil & - & - & 0.37 & 0.31 & -0.53 & - & 0.15 & 2.15 & -1.58 & [31] \\
\hline Huainan, China & Reclaimed soil & - & - & -0.17 & -0.15 & 1.01 & 0.58 & -0.09 & 0.96 & -1.17 & {$[32]$} \\
\hline Datong, China & Reclaimed soil & -2.16 & - & - & 0.08 & - & - & 0.07 & -1.22 & 1.19 & [33] \\
\hline Panyi, China & Reclaimed soil & -1.41 & - & - & -0.07 & - & - & 0.31 & -0.74 & 0.49 & [33] \\
\hline Xinzhuangzi, China & Reclaimed soil & -1.55 & - & - & 0.04 & - & - & 0.00 & -0.50 & 0.98 & [33] \\
\hline Baodian, China & Reclaimed soil & - & - & 0.01 & -0.45 & - & - & -0.17 & 1.45 & - & {$[34]$} \\
\hline Heidaigou, China & Reclaimed soil & -0.94 & - & -0.84 & -1.32 & -0.69 & 0.03 & -1.80 & -3.58 & -2.17 & [35] \\
\hline Huabei, China & & -1.01 & - & -0.55 & -1.10 & - & - & - & - & - & [36] \\
\hline Jilin Province, China & Top soil & - & - & -0.63 & -0.98 & -0.61 & - & -1.03 & -2.26 & - & {$[37]$} \\
\hline Dingji, China & Reclaimed soil & 0.04 & - & -1.12 & -0.50 & -1.37 & - & -0.84 & -2.47 & - & [38] \\
\hline Guizhou Province, China & Mine soil & - & - & 0.42 & - & - & - & -0.06 & - & - & [39] \\
\hline Raniganj, India & Native soil & - & -0.89 & -1.19 & -1.79 & -1.19 & -0.03 & -2.38 & 0.41 & - & [40] \\
\hline Surat, India & Agricultural Soil & - & - & 0.10 & 1.38 & -1.49 & 1.29 & -1.26 & 1.12 & - & [41] \\
\hline Raniganj, India & Top soil & - & -3.32 & 0.55 & 0.16 & 1.17 & 2.22 & - & - & - & [42] \\
\hline Sonepur Bazari, India & Opencast mine soil & - & 0.14 & -1.11 & -0.64 & -0.11 & 0.34 & -0.64 & -5.9 & - & [43] \\
\hline Jaintia, India & AMD contaminated soil & - & -1.88 & -0.91 & -0.89 & 0.43 & 0.52 & -0.89 & 1.09 & - & [44] \\
\hline Makum, India & AMD contaminated soil & - & -1.10 & -0.68 & -0.90 & 0.93 & 1.64 & -1.62 & 0.87 & - & {$[45]$} \\
\hline Ledo, Tinsukia, India & Mine soil & - & 0.11 & - & - & 0.07 & 1.71 & 2.1 & 1.85 & - & [46] \\
\hline Gangreung, Korea & AMD contaminated soil & - & - & -0.21 & -0.05 & -1.57 & 0.67 & -0.37 & 0.61 & - & [17] \\
\hline Barapukuria, Bangladesh & AMD contaminated soil & 0.03 & 1.14 & 1.55 & - & - & - & 3.35 & - & - & {$[8 \bullet]$} \\
\hline Barapukuria, Bangladesh & Paddy soils & 0.39 & 0.29 & 0.66 & -0.54 & 0.01 & 1.88 & 2.15 & - & - & [18] \\
\hline Tibagi River watershed, Brazil & Regular soils & - & 2.11 & - & - & - & - & - & 2.64 & - & {$[47 \bullet]$} \\
\hline Oltu, Turkey & & - & - & -1.50 & -0.85 & 0.35 & 1.16 & -0.32 & -4.58 & 2.94 & [48] \\
\hline Ptolemais, Greece & & -0.48 & - & & & -2.60 & -1.40 & & & - & [49] \\
\hline Ptolemais-Amynteon, Greece & Regular soil & -0.88 & - & -0.10 & 0.11 & 2.30 & 3.87 & 0.59 & -1.53 & - & {$[50]$} \\
\hline Ptolemais-Amynteon, Greece & Reclamed soil & -1.45 & - & -0.85 & -0.38 & 0.95 & 2.36 & 0.53 & -1.53 & - & {$[50]$} \\
\hline Douro, Portugal & Waste impacted soil & 1.16 & -2.62 & -0.83 & -0.21 & -0.20 & -0.32 & -0.47 & -1.85 & - & {$[51]$} \\
\hline Tula, Moscow, Russia & & 0.03 & - & 0.70 & - & - & - & - & - & - & {$[52]$} \\
\hline Palapye, Botswana & Mine soil & -0.80 & - & -0.64 & -0.26 & 0.24 & 0.83 & -0.90 & - & - & [53] \\
\hline Smolnica, Southern Polland & Reclaimed soil & - & - & 0.48 & -1.23 & & & -0.09 & 1.19 & - & {$[54]$} \\
\hline Emlahleni, South Arfica & Mine soil & -5.9 & - & -1.49 & -0.9 & 1.97 & -0.41 & -1.16 & - & - & {$[55]$} \\
\hline Okaba, Nigeria & Mine soil & - & - & -6.07 & -6.4 & - & -2.63 & -6.4 & -0.26 & - & {$[56]$} \\
\hline Pokrok, Czech Republic & Reclaimed soil & -1.93 & -0.5 & -2.00 & -1.6 & - & - & -1.2 & -1.41 & - & {$[57]$} \\
\hline Minimum & & -5.9 & -3.32 & -6.07 & -6.4 & -2.60 & -2.63 & -6.4 & -5.9 & -2.17 & \\
\hline Maximum & & 1.16 & 2.11 & 1.55 & 1.38 & 2.48 & 3.87 & 3.35 & 2.64 & 2.94 & \\
\hline
\end{tabular}

No. number of samples, $\mathrm{As}$ arsenic, $\mathrm{Mn}$ manganese, $\mathrm{Zn}$ zinc, $\mathrm{Cu}$ copper, $\mathrm{Cr}$ chromium, $\mathrm{Ni}$ nickel, $\mathrm{Pb}$ lead, $\mathrm{Cd}$ cadmium, $\mathrm{Hg}$ mercury - indicates data not available

value of Chinese soils. The highest concentration was observed from Barapukuria coal mine in Bangladesh, which was the only site to exceed the target limit of $85 \mathrm{mg} / \mathrm{kg}$ established for soil remediation [65].
The $I_{\text {geo }}$ value of $\mathrm{Pb}$ (-6.4 to 3.35) shows no contamination to strong contamination (Table 10). The $C F$ varies from 0.02 to 15.35 , indicating no contamination to very high contamination (Table 9). The $C F$ value for soils from Barapukuria, 
Bangladesh were $>6$, and have the highest $\mathrm{I}_{\text {geo }}$ value, indicating high contamination.

\section{Mercury (Hg)}

Mercury is a persistent, toxic, and bio-accumulative heavy metal. The concentration of $\mathrm{Hg}$ in soil in the vicinity of coal mines varies from 0.02 to $0.96 \mathrm{mg} / \mathrm{kg}$ (Table 8), the soils from Datong, Panyi, and Xinzhungzi in China and the Tibagi River wastershed in Brazil exceed the world average soil concentrations. These soils are considered to be enriched with $\mathrm{Hg}$ according to Gustin and Lindberg [69] since their concentrations exceeded $\geq 0.1 \mathrm{mg} / \mathrm{kg}$. The highest average concentration was recorded from the Tibagi River wastershed, and exceeds the optimum levels for $\mathrm{Hg}(0.3 \mathrm{mg} / \mathrm{kg})$ for clean soil according to the Dutch Guidelines [65]

The $I_{\text {geo }}$ value varies from -2.17 to 2.9 (Table 10), which fall under class 0 and class 3 , showing uncontaminated to moderately contaminated. Similarly, the $C F$ varies from 0.33 to 11.5 , which indicates low to very high contamination. The highest $C F$ and $I_{\text {geo }}$ values were found in Oltu, Turkey, indicating high contamination.

\section{Overall Metal Contamination in the Studied Soils}

The comprehensive state of contamination of the studied soils around various coal mines was evaluated based on four parameters: $C_{d e g}, m C_{d}, P L I$, and NIPI (Table 9). Around $60 \%$ of the sites in the studies reviewed show $P L I$ values $>1$, indicating soil pollution. The $C_{d e g}$ values of the studied soils vary from 1.52 to 35.9 , while the $m C d$ values varied from 0.25 to 7.8. Based on these indices, soil from Pindingshan, Xuzhou, and Boadian coal mines in China; Surat coal mine in India; Bhuiyan coal mine in Bangladesh; Ledo coal mines in India; and the Tibagi River watershed in Brazil have a high degree of pollution based on the concentrations of $\mathrm{Cd}, \mathrm{Pb}, \mathrm{Cu}, \mathrm{Cr}$, and Zn. Soils from Huainan, China; Jaintia and Makum, India; and Gangreung, Korea are categorized as having a moderate degree of contamination, and the rest are classified as having a low degree of contamination. This observation is consistents with NIPI values.

\section{Potential Ecological Risk Analysis}

The ecological risk of individual elements $\left(E_{i}\right)$ varied significantly among elements (Table 11 ): As, $0.43-33.5$; Zn, 0.024.3; $\mathrm{Cu}, 0.08-19.5$; Cr, 0.09-16.7; Pb, 0.73-76.7; Cd, 0.73274; and $\mathrm{Hg}, 13-460$. In terms of the average values of $E_{i}$, these metals were arranged in the following descending order: $\mathrm{Hg}>\mathrm{Cd}>\mathrm{As}>\mathrm{Pb}>\mathrm{Cu}>\mathrm{Cr}>\mathrm{Mn}$. $\mathrm{Hg}$ and $\mathrm{Cd}$ pose a high risk for soil bodies, their maximum values reaching 469 and 274 , respectively. Five sites have a strong potential ecological risk for $\mathrm{Hg}$, and ten sites have a strong potential ecological risk from $\mathrm{Cd}$, whereas other trace elements only show a slight ecological risk to the environment (Table 11). This assessment indicates $\mathrm{Hg}$ and $\mathrm{Cd}$ are the key elements to be further studied and considered as important in the assessment of metals in coal mine soils. With regard to $\mathrm{Cd}$, the Xuzhou and Baodian coal fields of China, Ledo coal mines in India, Tibagi River watershed in Brazil, and Smolnica coal mines in southern Poland show a high degree of ecological risk. With regard to $\mathrm{Hg}$, a high degree of pollution was observed at Datong and Xinzhuangzi in China, and Oltu in Turkey.

The potential ecological risk $(R I)$ represents the sensitivity of various biological communities to toxic substances and illustrates the potential ecological risk caused by overall metal contamination. Based on the RI values (Table 11), soil from Xuzhou (China), Tibagi River (Brazil), Smolnica coal mines in southern Poland, and Oltu (Turkey) shows a very high risk of contamination.

\section{Management of Soil Contamination}

Management of soil contamination is a global concern for environmental and agricultural sustainability. This can be classified into immediate and long-term actions as follows:

\section{Immediate Action}

Immediate action may involve formulating soil quality guidelines and standards, conducting monitoring programs, and enforcing environmental regulations. Government regulators and coal-mining companies must take the first steps to monitor metal pollution nearby the mining areas. The most effective step in managing soil pollution is to control contaminants from their sources, especially waste discharge and overburden, and enforcement of strong environmental regulations and laws $[70,71]$.

\section{Long-term Action}

Long-term measures may include concluding an Environmental Impact Assessment (EIA) on existing and proposed coalmining projects, and utilizing appropriate technology for remediation of toxic metals. There are several physical, chemi$\mathrm{cal}$, and biological remedial technologies that have been developed to manage soil pollution [72, 73] (Fig. 3).

\section{Physical Methods}

Physical technologies mainly include soil replacement/ mixing, capping, and thermal desorption. Soil replacement means using clean soil to replace the contaminated soils with the aim of diluting the metals [73, 74]. Soil capping is a reliable technology that does not involve removal of contaminated soils. Although these traditional methods can 
Table 11 Ecological risk $\left(E_{i}\right)$ and potential ecological risk factor $(R I)$ of trace elements in soils worldwide

\begin{tabular}{|c|c|c|c|c|c|c|c|c|c|c|}
\hline Location & Soil type & As & $\mathrm{Zn}$ & $\mathrm{Cu}$ & $\mathrm{Cr}$ & $\mathrm{Pb}$ & $\mathrm{Cd}$ & $\mathrm{Hg}$ & RI & Reference \\
\hline Antaibao, China & Reclaimed soil & - & - & 5.52 & 1.68 & 1.72 & 5.51 & 66.67 & 81.10 & {$[26]$} \\
\hline Fushun, China & Reclaimed soil & - & 0.68 & 2.88 & 1.21 & - & 25.71 & - & 30.48 & {$[27]$} \\
\hline Heidaigou, China & Reclaimed soil & - & 0.83 & 3.20 & 2.08 & 2.19 & 5.51 & 20.00 & 33.81 & {$[28]$} \\
\hline Laohutai, China & Reclaimed soil & - & 0.97 & 6.09 & 2.04 & - & 1.22 & - & 10.32 & [27] \\
\hline Pindingshan, China & Reclaimed soil & - & 0.59 & 6.91 & 16.76 & 10.82 & 79.59 & - & 114.67 & [29] \\
\hline Pingshuo, China & Reclaimed soil & - & - & - & 1.15 & 4.08 & 12.86 & 13.33 & 31.42 & {$[30]$} \\
\hline Xuzhou, China & Reclaimed soil & - & 1.93 & 9.27 & 2.07 & 8.40 & 195.92 & 20.00 & 237.60 & {$[31]$} \\
\hline Huainan, China & Reclaimed soil & - & 1.33 & 6.77 & 6.02 & 7.09 & 85.71 & 26.67 & 133.60 & {$[32]$} \\
\hline Datong, China & Reclaimed soil & 3.35 & - & 7.93 & - & 7.93 & 18.98 & 136.67 & 174.87 & {$[33]$} \\
\hline Panyi, China & Reclaimed soil & 5.65 & - & 7.14 & - & 9.38 & 26.33 & 84.00 & 132.50 & [33] \\
\hline Xinzhuangzi, China & Reclaimed soil & 5.14 & - & 7.72 & - & 7.55 & 31.22 & 118.00 & 169.63 & {$[33]$} \\
\hline Baodian, China & Reclaimed soil & - & 1.50 & 5.48 & - & 6.70 & 120.61 & - & 134.29 & {$[34]$} \\
\hline Heidaigou, China & Reclaimed soil & 7.80 & 0.84 & 3.01 & 1.86 & 2.16 & 3.67 & 13.33 & 32.68 & {$[35]$} \\
\hline Huabei, China & & 7.43 & 1.02 & 3.51 & - & - & - & - & 11.96 & {$[36]$} \\
\hline Jilin Province, China & Top soil & - & 0.96 & 3.81 & 1.96 & 3.71 & 9.18 & - & 19.63 & {$[37]$} \\
\hline Dingji, China & Reclaimed soil & 15.44 & 0.69 & 5.32 & 1.16 & 4.22 & 7.96 & - & 34.78 & {$[38]$} \\
\hline Guizhou, China & Mine soil & - & 2.0 & - & - & 7.5 & - & - & 9.5 & [39] \\
\hline Raniganj, India & Native soil & - & 0.65 & 2.16 & 1.32 & 1.45 & 58.47 & - & 64.06 & {$[40]$} \\
\hline Surat, India & Agricultural Soil & - & 1.60 & 19.57 & 1.07 & 3.16 & 95.51 & - & 120.91 & {$[41]$} \\
\hline Raniganj, India & Top soil & - & 2.19 & 8.39 & 6.77 & - & - & - & 17.35 & {$[42]$} \\
\hline Sonepur Bazari, India & Opencast mine & 3.4 & 0.69 & 4.7 & 2.7 & 4.8 & 0.73 & - & 17.2 & [43] \\
\hline Jaintia, India & AMD contaminated soil & - & 0.79 & 4.06 & 4.03 & 4.08 & 93.67 & - & 106.64 & {$[44]$} \\
\hline Makum, India & AMD contaminated soil & - & 0.94 & 4.02 & 5.70 & 2.46 & 80.82 & - & 93.94 & {$[45]$} \\
\hline Ledo, Tinsukia, India & Mine soil & - & - & - & 3.1 & 32.4 & 159.18 & - & 194.7 & {$[46]$} \\
\hline Gangreung, Korea & AMD contaminated soil & - & 1.29 & 7.27 & 1.01 & 5.83 & 67.35 & - & 82.75 & [17] \\
\hline Barapukuria, Bangladesh & AMD contaminated soil & 15.35 & 4.37 & & & 76.77 & - & - & 96.49 & {$[8 \bullet]$} \\
\hline Barapukuria, Bangladesh & Paddy soils & 19.65 & 2.36 & 5.14 & 3.02 & 33.44 & - & - & 63.61 & {$[18]$} \\
\hline Tibagi River watershed, Brazil & Regular soils & - & - & - & - & - & 274.29 & - & 274.29 & {$[47 \bullet]$} \\
\hline Oltu, Turkey & & - & 0.53 & 4.15 & 3.83 & 6.06 & 1.84 & 460.00 & 476.40 & [48] \\
\hline Ptolemais, Greece & & 10.79 & - & - & 0.49 & - & - & - & 11.28 & [49] \\
\hline Ptolemais-Amynteon, Greece & Regular soil & 8.16 & 1.39 & 8.10 & 14.74 & 11.38 & 15.31 & - & 59.08 & {$[50]$} \\
\hline Ptolemais-Amynteon, Greece & Reclamed soil & 5.48 & 0.83 & 5.76 & 5.81 & 10.87 & 15.31 & - & 44.05 & {$[50]$} \\
\hline Douro, Portugal & Waste-impacted soil & 33.60 & 0.84 & 6.47 & 2.60 & 5.46 & 12.24 & - & 61.22 & [51] \\
\hline Tula, Moscow, Russia & & 15.26 & 2.43 & - & - & - & - & - & 17.69 & {$[52]$} \\
\hline Palapye, Botswana & Mine soil & 8.60 & 0.96 & 6.28 & 3.53 & 4.04 & - & - & 23.41 & {$[53]$} \\
\hline Smolnica, Southern Polland & Reclaimed soil & - & 2.09 & 3.19 & - & 7.0 & 101 & - & 113.3 & {$[54]$} \\
\hline Emlahleni, South Africa & Mine soil & 0.43 & 0.53 & 3.9 & 11.8 & 3.36 & - & - & 20 & {$[55]$} \\
\hline Okaba, Nigeria & Mine soil & - & 0.02 & 0.08 & - & 0.08 & 36.7 & - & 36.9 & {$[56]$} \\
\hline Pokrok, Czech Republic & Reclaimed soil & 3.9 & 0.37 & 2.4 & - & 3.26 & 16.5 & - & 26.5 & {$[57]$} \\
\hline Minimum & & 0.43 & 0.02 & 0.08 & 0.49 & 0.08 & 0.73 & 13.33 & 10.32 & \\
\hline Maximum & & 33.60 & 4.37 & 19.57 & 16.76 & 76.77 & 274.29 & 460.00 & 476.4 & \\
\hline
\end{tabular}

No. number of samples, $\mathrm{As}$ arsenic, $\mathrm{Mn}$ manganese, $\mathrm{Zn}$ zinc, $\mathrm{Cu}$ copper, $\mathrm{Cr}$ chromium, $\mathrm{Ni}$ nickel, $\mathrm{Pb}$ lead, $\mathrm{Cd}$ cadmium, $\mathrm{Hg}$ mercury - indicates data not available

effectively isolate the contaminated soil or reduce the contamination levels, they are high cost technologies, move contamination to another area, and are not suitable for large areas $[75,76]$. Another physical technology is thermal desorption, which is based on the phenomenon of the pollutant's volatility upon heating the contaminated soil. Although this technique can be applied for volatile elements such as $\mathrm{Hg}$ and As, application is limited 
Fig. 3 Various physical, chemical, and biological methods for remediation of heavy metals in soils

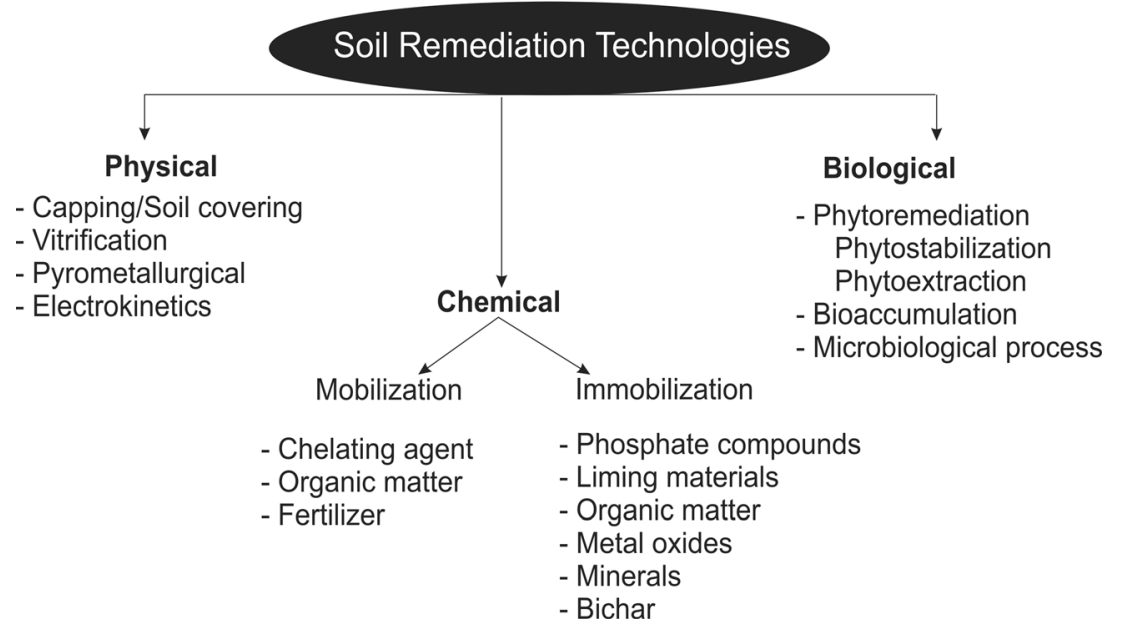

because of the need for expensive heating devices and a long-term desorption time in the remediation process [73].

Another technology is electrokinetics, which is a new remediation technique based on the phenomenon of contaminant migration in the form of charged species in an electric field [77]. The current is applied by inserting electrodes in the contaminated soils. Then under the influence of an electromagnetic field, contaminants migrate through the soil within the cathode or anode area, where they can be removed by a variety of processes, including electroplating at the electrodes or chemical precipitation/co-precipitation at the electrodes [78]. However, this technique is strongly dependent on soil conductivity because energy consumption is directly related to the conductivity of soil between the electrodes. Also, this remediation technique may not feasible due to its high cost [79].

\section{Chemical Methods}

This technique is based on two fundamental processes to remediate soils, chemical leaching and chemical fixation (immobilization) [80]. Chemical leaching is washing of the contaminated soil by using reagents such as acids, salts, chelating agents, surfactants, etc. The soil washing cost is largely dependent on the extent to which contaminated sites are processed. Use of strong acid washing leads to decreases in soil productivity and adverse changes in the chemical and physical structure of soils due to mineral dissolution [81]. For chelation, EDTA can work in a wide $\mathrm{pH}$ range and could extract a significant fraction of metals from contaminated soils [82]. But EDTA is very stable in the soil and can make soil unfit for further use because residual EDTA can slowly leach nutrients from the soil and can disturb the physical and chemical properties of soil. Also, EDTA is expensive and its biological degradability is different. As an alternative, current research has focused on biodegradable and organic chelates such as saponin and tea saponin, which effectively removed toxic metals from soils and greatly reduced the environmental risk
$[83,84]$. Furthermore, it has been found that some low molecular weight organic acids such as citric and tartaric acid could solubilize metals from contaminated soil through complexation reactions [85, 86]. This is inexpensive, biodegradable, and less destructive to soil structure compared to EDTA. However, this technology is not a permanent solution because it needs long-term monitoring [86]. Chemical treatments can be performed both ex situ and in situ. However, in situ chemical agents must be carefully chosen so they do not cause further contamination. The major problem associated with chemical treatment is the nonspecific nature of the chemical reagents. The chemical added to treat one metal can also target reactive metals and can make them more toxic or mobile. Moreover, the remediation of polluted soil containing trace elements is technically difficult because of high costs and other effects. In addition, these approaches have been mostly studied at a laboratory level, though some are being studied in the field, but they are still small scale.

\section{Biological Methods}

Recently, use of phytoremediation as a potentially promising, low-cost, and in-situ new technology to remove pollutants from contaminated soils has gained increasing attention as an alternative to conventional physical and chemical methods [87, 88]. Phytostabilization and phytoextraction are the main two types of phytoremediation methods to treat metal-contaminated soils [89]. The key of these methods is the selection of appropriate plant species that are tolerant to trace elements. Although this method is being field-tested at a variety of sites in the USA and Europe, full-scale application of these techniques is limited and few performance data are available. Phytoremediation methods will likely be limited to use in shallow soils with low levels of metal contamination. Although phytoremediation is currently receiving more advantages over other physico-chemical 
methods, there are still some issues associated with this technique. For example, this method may not be applicable in areas of elevated contamination, as plants could be affected by metal toxicity. In addition, to date this technique has only been tested in laboratories, and more research is required in the field.

Overall, metal contamination in soils varies considerably depending on site-specific factors, especially those that affect the mobility of metals. Thus, the selection of suitable techniques depends on the soil type, extent and nature of the metal contamination, cost and availability of materials, and relevant regulations.

\section{Conclusions}

This paper reviews the total concentration of trace elements (As, $\mathrm{Cd}, \mathrm{Cu}, \mathrm{Cr}, \mathrm{Hg}, \mathrm{Mn}, \mathrm{Ni}, \mathrm{Pb}$, and $\mathrm{Zn}$ ) in soils near coal mines in various countries. The average concentration of these elements varies widely, but most of these values are higher than their respective values in world background soils. The $I_{g e o}$ and $C F$ values indicate that the contamination levels of $\mathrm{Ni}$ and $\mathrm{Pb}$ are higher than other elements. The highest $I_{g e o}$ values for Ni are observed in Ptolemain-Amynteaon, Greece, while the highest values for $\mathrm{Pb}$ were recorded at Barapukuria, Bangladesh. The highest $I_{\text {geo }}$ values of $\mathrm{As}, \mathrm{Hg}, \mathrm{Cd}$, and $\mathrm{Cr}$ were found at the Tula mines in Russia, Oltu in Turkey, Barapukuria in Bangladesh, and Pindingshan in China, respectively. This is consistent with the $C F$ index. This clearly shows that individual metal pollution in soils around the coal mines is site-specific. The ecological risk index $\left(E_{i}\right)$ indicates an obvious risk from $\mathrm{Cd}$, especially in the Tabagi River watershed (Brazil) and Ledo coal mines (India), and $\mathrm{Hg}$, especially in the Oltu coal-mining area (Turkey), and therefore are chosen as the key elements to predict pollution trends. The integrated indices, such as $C_{d e g}, m C_{d}, I P L$, and NIPI, reveal that the soils from Barapukuria (Bangladesh), Ptolemais-Amynteon (Greece), and the Tibagi River (Brazil) have a higher degree of contamination than other sites.

To control metal contamination in soil, monitoring and legislative measures must be taken as immediate steps. For the long term, scientific research and remedial technology should be implemented. Chemical immobilization, soil leaching, and phytoremediation are frequently used for remediation purposes; among them phytoremediation is the best available technology for remediation of soils. However, these technologies have mainly been demonstrated at an experimental level and more work should be focused at field level.

\section{Compliance with Ethical Standards}

Human and Animal Rights and Informed Consent No Human or Animal Studies Performed by the Authors:

This article does not contain any studies including human or animal subjects.

Human Studies Done by Authors (but no Animal Studies) This article does not contain any studies with animal subjects performed by any of the authors. With regard to the authors' research cited in this paper, all procedures were followed in accordance with the ethical standards of the responsible committee on human experimentation and with the Helsinki Declaration of 1975, as revised in 2000 and 2008.

Animal Studies Done by Authors (but no Human Studies) This article does not contain any studies with human subjects performed by any of the authors.

With regard to the authors' research cited in this paper, all institutional and national guidelines for the care and use of laboratory animals were followed.

\section{References}

Paper of particular interest, published recently, have been highlighted as:

- Of importance

1. Elliott MA. Chemistry of coal utilization: second supplementary colume. New York, United States: John Wiley and Sons; 1981.

2. WCA (World Coal Association) Coal statistics report. http://www. worldcoal.org/resources/coal-statistics/. 2013; Sourced on 3/11/14.

3. WEC (World Energy Resources) Summary report. www. worldenergy.org/wp-content/uploads/2013/10/WEC_Resources summary-final.pdf. 2013; Sourced on 20/11/14.

4. Equeenuddin Sk Md, Tripathy S, Sahoo PK, Panigrahi MK. Hydrogeochemical characteristics of acid mine drainage and water pollution at Makum Coalfield, India. J Geochem Expl. 2010;3:7582.

5. Chuncai Z, Guijian L, Dun W, Ting F, Ruwei W, Xiang F. Mobility behavior and environmental implications of trace elements associated with coal gangue: a case study at the Huainan Coalfield in China. Chemosphere. 2014;95:193-9.

6. Sahoo PK, Tripathy S, Equeenuddin Sk Md, Panigrahi MK. Geochemical characteristics of coal mine discharge vis-à-vis behavior of rare earth elements at Jaintia Hills Coalfield, Northeastern India. J Geochem Expl. 2012;112:235-243.

7. Banat KM, Howari FM, Al-Hamada AA. Heavy metals in urban soils of central jordan: should we worry about their environmental risks? Environ Res. 2005;97:258-73.

8. Bhuiyan MAH, Parvez L, Islam MA, Dampare SB, Suzuki S. Heavy metal pollution of coal mine-affected agricultural soils in the northern part of Bangladesh. J Hazard Mater. 2010;173:384 92. The source of metals has been well explained.

9. Sahoo PK, Tripathy S, Equeenuddin SM, Panigrahi MK. Influence of different forms of acidities on soil microbiological properties and enzyme activities at an acid mine drainage contaminated site. J Hazard Mater. 2010;179:966-75.

10. Wuana RA, Okieimen FE. Heavy metals in contaminated soils: a review of sources, chemistry, risks and best available strategies for remediation. ISRN Ecology 2011. ID 402647. 
11. Zhang ZY, Abuduwaili J, Jiang FQ. Determination of occurrence characteristics of heavy metals in soil and water environments in tianshan mountains, central Asia. Anal Lett. 2013;46:2022-31.

12. Rashid H, Shanto MAS, Roy DR, Hossain MS, Islam MS, Hoque MMM, et al. Impact of coal mining on soil, water and agricultural crop production: a cross-sectional study on Barapukuria coal mine industry, Dinajpur, Bangladesh. J Environ Sci Res. 2014;1(1): 0000001 . This coalmine has significant impact on soil quality, mainly contamination of heavy metals.

13. Nagajyoti PC, Lee KD, Sreekanth TVM. Heavy metals, occurrence and toxicity for plants: a review. Environ Chem Lett. 2010;8:199216.

14. Seshan BRR, Natesan U, Deepthi K. Geochemical and statistical approach for evaluation of heavy metal pollution in core sediments in southeast coast of India. Int J Environ Sci Technol. 2010;7(2): 291-306.

15. Gao H, Bai J, Xiao R, Liu P, Jiang W, Wang J. Levels, sources and risk assessment of trace elements in wetland soils of a typical shallow freshwater lake, China. Stoch Environ Res Risk A. 2013;27: 275-84.

16. Zhuang W, Gao X. Integrated assessment of heavy metal pollution in the surface sediments of the Laizhou Bay and the coastal waters of the Zhangzi Island, China: comparison among typical marine sediment quality indices. PLoS ONE. 2014;9:e94145.

17. Kim JY, Chon HY. Pollution of a water course impacted by acid mine drainahe in the Imgok creek of the Gangreung coal field, Korea. Appl Geochem. 2001;16:1387-96.

18. Halim MA, Majumdar RK, Zaman MN. Paddy soil heavy metal contamination and uptake in rice plants from adjecent area Barapukuria coal mine, Northwest, Bangladesh. Arab J Geosci. 2014. doi:10.1007/s12517-014-1480-1.

19. Hakanson L. An ecological risk index for aquatic pollution control, a sedimentological approach. Water Res. 1980;14:975-1001.

20. Muller G. Index of Geoaccumulation in sediments of the Rhine River. Geol J. 1969;2:108-18.

21. Loska K, Wiechula D, Korus I. Metal contamination of farming soils affected by industry. Environ Int. 2004;30:159-65.

22. Machender G, Dhakate R, Prasanna L, Govil PK. Assessment of heavy metal contamination in soils around Balanagar industrial area, Hyderabad, India. Environ Earth Sci. 2011;63:945-53.

23. Abrahim GMS, Parker RJ. Assessment of heavy metal enrichment factors and the degree of contamination in marine sediments from Tamaki Estuary, Auckland, New Zealand. Environ Monit Assess. 2008; 136:227-38.

24. Tomilson DC, Wilson DJ, Harris CR, Jeffrey DW. Problem in assessment of heavy metals in estuaries and the formation of pollution index. Helgoländer Meeresun. 1980;33(1-4):566-75.

25. Yang ZP, Lu WX, Long YQ, Bao XH, Yang QC. Assessment of heavy metals contamination in urban topsoil from Changchun City, China. J Geochem Explor. 2011;108:27-38.

26. Fan WH, Bai ZK, Li HF, Qiao JY, Xu JW, Li X. Potential ecological assessment of heavy metals in reclaimed soils. Trans CSAE. 2011;27(1):348-54.

27. Yu J, Liu J, Wang J, Li Z, Zhang X. Spatial-temporal variation of heavy metal elements content in covering soil of reclamation area in Fushun coal mine. Chinese Geogr Sci. 2002;12:268-72.

28. Ma J, Zhang S, Yao H, Wan J. Temporal cumulative effects of heavy metals and metalloid elements in covering soil of opencast coal mine reclamation area. J Arid Land Res Environ. 2012;26:6974 (In Chinese).

29. Wang Z, Ma J, Geng P, Gu L. Heavy metals distribution and pollution of the reclaimed soil in the subsidence area of Pingdingshan city coal mine. J Agro Environ Sci. 2009;28(4):668-72 (in Chinese).

30. Ge Y, Cui X, Bai Z. Evaluation on potential ecological risk of heavy metals pollution in reclaimed soil of opencast-taking Pingshuo opencast mine as an example. J Shanxi Agric Univ (Natural Science Edition). 2008;28:85-8. In Chinese.

31. Wang Y, Dong J. Potential ecological risk assessment of filling reclaimed soils polluted by heavy metals in mine area. J China Coal Soc. 2009;34(5):650-5 (in Chinese).

32. Niu S, Gao L, Zhao J. Distrubution and risk assessment of heavy metals in the Xinzhuangzi reclamation soil from the Huainan coal mining area, China. Hum Ecol Risk Assess. 2015;0:1-13.

33. Yao D, Jun M, Zhang Z. Heavy metal pollution and potential ecological risk in reclaimed soils in Huainan mining area. J Coal Sci Eng. 2010;16:316-9.

34. Jing Y, Gao B, Ma Z. Potential ecological risk assessment of soil heavy metals contamination around coal gangue piles of Baodian coal mine area of Shandong, China. IEEE. 2011.

35. Wang J, Liu W, Yang R, Zhang L, Ma J. Assessment of the potential ecological risk of heavy metals in reclaimed soils at an opencast coal mine. Disaster Adv. 20136 (S3).

36. Shi GL, Lou LQ, Zhang S, Xia XW, Cai QS. Arsenic, copper, and zinc contamination in soil and wheat during coal mining, with assessment of health risks for the inhabitants of Huaibei, China. Environ Sci Pollut Res. 2013;20:8435-45.

37. Jiang X, Lu WX, Zhao HQ, Yang QC, Yang ZP. Potential ecological risk assessment and prediction of soil heavy-metal pollution around coal gangue dump. Nat Hazards Earth Syst Sci. 2014;14: $1599-610$

38. You M, Huang Y, Lu J, Li C. Characterization of heavy metals in soil near coal mines and a power plant in Huainan, China. Anal Lett. 2014. doi:10.1080/00032719.2014.940531.

39. Chen Y, Zhao HX, Xie ZH, Huang HY, Zang SY, Lian B. Heavy metal pollution characteristics in the Kaili coal mining region, Guizhou Province, China. J Residual Sci Technol. 2015;12:S12309 .

40. Sadhu K, Adhikari K, Gangopadhyay A. Assessment of heavy metal contamination of soils in and around open cast mines of Raniganj Area, India. Int J Environ Eng Res. 2012;1:77-85.

41. Ladwani KD, Ladwani KD, Manik VS, Ramteke D, Assessment of heavy metal contaminated soil near coal mining area in Gujarat by toxicity characteristics leaching procedure. Int J Life Sc Bt Pharm Res. 2012.

42. Das SK, Chakrapani GJ. Assessment of trace metal toxicity in soils of Raniganj Coalfield, India. Environ Monit Assess. 2011;177:6371.

43. Masto RE, Sheik S, Nehru G, Selvi VA, George J, Ram LC. Assessment of environment soil quality around Sonepur Bazari mine of Raniganj coalfield, India. Solid Earth. 2015;6:811-21.

44. Sahoo PK. Geochemical appraisal of acid mine drainage around Jaintia Hills coalfield, Meghalaya, India, Unpublished PhD Thesis, Indian Institute of Technology, Kharagpur; 2011.

45. Equeenduddin Sk.Md. Control of coal and overburden on acid mine drainage and metal mobilization at Makum coalfield, Assam, India, Unpublished $\mathrm{PhD}$ Thesis, Indian Institute of Technology, Kharagpur; 2010.

46. Reza SK, Baruah U, Singh SK, Das TH. Geostatistical and multivariate analysis of soil heavy metal contamination near coal mining area. Northeastern India. Environ Earth Sci. 2015;73:5425-33.

47. Galunin E, Ferreti J, Zapelini I, Vieira I, Tarley CRT, Abrao T, et al. Cadmium mobility in sediment and soils from coal mining area on Tibagi River watershed: environmental risk assessment. J Hazard Mater. 2014;265:280-7. Amongst the studied literature, this paper reported highest concentrations of $\mathrm{Cd}$ and $\mathrm{Mn}$ in coal mine soils.

48. Tozsin G. Hazardous elements in soil and coal from the Oltu coal mine district, Turkey. Int J Coal Geol. 2014;131:1-6.

49. Modis K, Vatalis KI, Sachanidis C. Spatiotemporal risk assessment of soil pollution in a lignite mining region using a Bayesian maximum entropy (BME) approach. Int J Coal Geol. 2013;112:173-9. 
50. Pentari D, Typou J, Goodarzi F, Foscolos AE. Comparision of elements of environmental concern in regular and reclaimed, near abandoned coal mines Ptolemais-Amynteon, northern Greece: impact on wheat crops. Int J Coal Geol. 2006;65:51-8.

51. Ribeiro J, Silva EF, Ward LC, Flores D. Petrographic, mineralogical and geochemical characterization of the Serrinhal coal waste pile (Douro Coalfield, Portugal) and the potential environmental impacts on soil, sediments and surface waters. Int J Coal Geol. 2010;83:456-66.

52. Komnitsas K, Modis K. Soil risk assessment of As and Zn contamination in a coal mining region using geostatistics. Sci Total Environ. 2006;371:190-6.

53. Zhai M, Totolo O, Modisi MP, Finkelman RB, Kelesite SM, Menyatso M. Heavy metal distribution in soils near Palapye, Botswana: an evaluation of the environmental impact of coal mining and combustion on soils in a semi-arid region. Environ Geochem Health. 2009;31:759-77.

54. Pietrzykowski M, Socha J, van Doorn NS. Linking heavy metal bioavailability $(\mathrm{Cd}, \mathrm{Cu}, \mathrm{Zn}$ and $\mathrm{Pb})$ in Scots pine needles to soil properties in reclaimed mine area. Sci Total Environ. 2014; 470471:501-510.

55. Maya M, Musekiwa C, Mthembi P, Crowley M. Remote sensing and geochemistry techniques for the assessment of coal mining pollution, Emalahleni (Witbank), Mpumalanga. S Afr J Geomatic 2015; 4: No 2.

56. Ameh EG. Multivariate statistical analysis and enrichment of heavy metal contamination of soil around Okaba coal mines. Am Eurasian J Agron. 2013;6:09-18.

57. Gholizadeh A, Boruvka L, et al. Estimation of potentially toxic elements contamination in anthropogenic soils on a Brown coal mining dumpsite by reflectance spectroscopy: a case study. PLoS ONE 10(2): e0117457.

58. Kabata-Pendias A, Pendias H. Trace elements in soils and plants. Boca Raton, FL: CRC Press; 1992.

59. Berrow ML, Reaves GA. Background levels of trace elements in soils. p. 333-340. In Proc. 1st Int. Conf. on Environmental Contamination. CEP Consultants. Edinburgh, Scotland. 1984.

60. Wedephol KH. The composition of the continental crust. Geochim Cosmochim Acta. 1995;59:1232-77.

61. Chen J, Wei F, Zheng C, Wu Y, Adriano DC. Background concentrations of elements in soils of China. Water Air Soil Pollut. 1991;57-58:699-712.

62. Shacklette HT, Boerngen JG. Element concentrations in soils and other surficial materials of the conterminous United States. USGS Professional Paper 1270. 1984.

63. Pérez C, Martínez MJ, Vidal J, Navarro C. Proposed reference values for heavy metals in calcaric fluvisols of the Huerta de Murcia (SE Spain). In: Sustainable use and management of soils in arid and semiarid regions (Fáz A., Ortiz R., Mermut A.R., eds). Quaderna Editorial, Cartagena, Murcia, Spain, 2002; 495-496.

64. Fadigas FS, Sobrinho NMB, Mazur N, Anjos LHC. Estimation of reference values for cadmium, cobalt, chromium, copper, nickel, lead, and zinc in brazilian soils. Commun Soil Sci Plant Anal. 2006;37:945-59.

65. VROM. Circular on target values and intervention values for soil remediation. Annex A. In: Dutch ministry of housing spatial planning and environment (VROM) (Ed.), The Hague, The Netherlands. 2000.

66. CCME (Canadian Council of Ministers of the Environment), Soil Quality Guidelines. 1991.

67. U.S. Environmental Protection Agency (USEPA). Integrated Risk Information System (IRIS) on Arsenic. National Center for Environmental Assessment, Office of Research and Development, Washington, DC; 1998.
68. Ajmone-Marsan F, Biasioli M. Trace elements in soils of urban areas. Water Air Soil Pollut. 2010;213:121-43.

69. Gustin MS, Lindberg SE. Assessing the contribution of natural sources to the global mercury cycle: the importance of intercomparing dynamic flux measurements. Fresenius J Anal Chem. 2000;366:417-22.

70. Chen HM, Zheng CR, Tu C, Zhu YG. Heavy metal pollution in soils in China: status and countermeasures. Ambio 1999:130-4.

71. Li Z, Ma Z, Kuijp TJ, Yuan Z, Hunag L. A review of soil heavymetal pollution from mines in China: pollution and health risk assessment. Sci Total Environ. 2014;468-469:843-53.

72. Shen ZG, Chen HM. Bioremediation of heavy metal polluted soils. Rural Ecol Environ. 2000;16(2):39-44.

73. Yao Z, Li J, Yu C. Review on remediation technologies of soil contaminated by heavy metals. Procedia Environ Sci. 2012;16: 722-9.

74. Zhang YF, Sheng JC, Lu QY. Review on the soil remediation technologies. Gansu Agric Sci Technol. 2004;10:36-8.

75. Mulligan CN, Yong RN, Gibbs BF. Remediation technologies for metal-contaminated soils and groundwater: an evaluation. Eng Geol. 2001;60:193-207.

76. Zhou DM, Hao XZ, Xue Y, et al. Advances in remediation technologies of contaminated soils. Ecol Environ Sci. 2004;13(2):234-42.

77. Luo QS, Zhang XH, Wang H, et al. Mobilization of 2, 4dichlorophenol in soils by non-uniform electrokinetics. Acta Sci Circumst. 2004;24(6):1104-9.

78. Smith LA, Means JL, Chen A, Alleman B, Chapma CC, Tixir JJS, et al. Remediation option for metal-contaminated sites. Boca Raton, FL: Lewis; 1995.

79. Evanko CR, Dzombak DA. Remediation of metal-contaminated soils and groundwater. Technology Evaluation Report, TE-97-01. Ground-Water Remediation Technologies Analysis Center, Pittsburg, PA. 1997.

80. Bolan N, Kunhikrishnan A, Thangarajan R, Kumpiene J, Park J, Makino T, et al. Remediation of heavy metal(loids) contaminated soils - to mobilize or to immobilize? J Hazard Mater. 2014;266: 141-66.

81. Reed BE, Carriere PC, Moore RJ. Flushing of $\mathrm{Pb}$ (II) contaminated soil using $\mathrm{HCl}$, EDTA, and $\mathrm{CaCl}_{2}$. Environ Eng. 1996;122:48-50.

82. Sun B, Zhao FJ, Lombi E, McGrath SP. Leaching of heavy metals from contaminated soils using EDTA. Environ Pollut. 2001;113: $111-20$.

83. Hong KJ, Tokunaga S, Kajiuchi T. Evaluation of remediation process with plant-derived biosurfactant for recovery of heavy metals from contaminated soils. Chemosphere. 2002;49(4):379-87.

84. Li GD, Zhang ZW, Jing P, et al. Leaching remediation of heavy metal contaminated fluvio-aquatic soil with tea-saponin. T Chinese Soc Agric Eng. 2009;25:231-5.

85. Wassy SA. Bioremediation of soils polluted by heavy metals using organic acids. PhD Thesis, McGill University, Montreal, Canada; 1998.

86. Ke X, Li P, Zhou Q, Yun Z, Sun T. Removal of heavy metals from a contaminated soil using tartaric acid. J Environ Sci. 2006;18:72733.

87. Lone MI, He Z, Stoffella PJ, Yang X. Phytoremediation of heavy metal polluted soils and water: progress and perspectives. J Zhejiang Univ Sci B. 2008;9:210-20.

88. Marques APGC, Rangel ASS, Castro PML. Remediation of heavy metal contaminated soils: phytoremediation as a potentially promising clean-up technology. Crit Rev Environ Sci Technol. 2009;39: $622-54$.

89. Wong $\mathrm{MH}$. Ecological restoration of mine degrade soils, with emphasis on metal contaminated soils. Chemosphere. 2003;50:775-80 